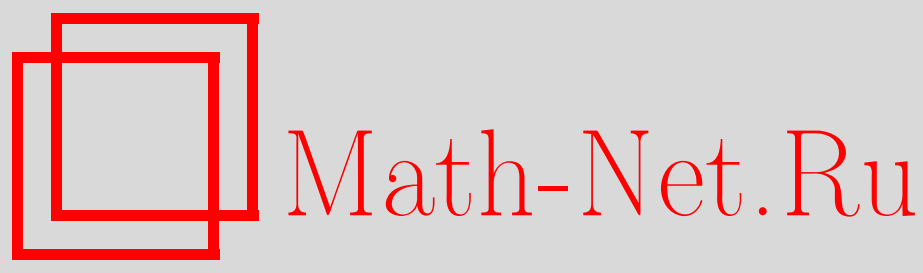

И. Кишимото, Т. Такахаши, Численный расчет калибровочных инвариантов для решений в $a$-калибровке в теории поля открытых струн, ТМФ, 2010, том 163, номер 3, 372-380

DOI: https://doi.org/10.4213/tmf6507

Использование Общероссийского математического портала Math-Net.Ru подразумевает, что вы прочитали и согласны с пользовательским соглашением http://www . mathnet.ru/rus/agreement

Параметры загрузки:

IP : 54.164 .48 .24

26 апреля 2023 г., 13:01:34

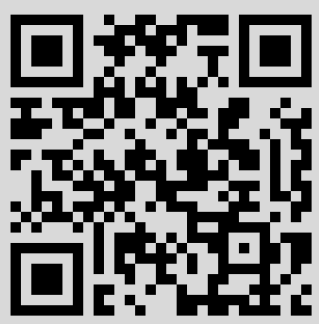




\title{
ФИЗИКА
}

Том 163, № 3

июнь, 2010

\section{ЧИСЛЕННЫЙ РАСЧЕТ КАЛИБРОВОЧНЫХ ИНВАРИАНТОВ ДЛЯ РЕШЕНИЙ В $a$-КАЛИБРОВКЕ В ТЕОРИИ ПОЛЯ ОТКРЫТЫХ СТРУН}

\begin{abstract}
В кубичной теории поля открытых бозонных струн вычислены калибровочные инварианты (действие и калибровочно-инвариантное перекрытие) для численных решений, удовлетворяющих условию $a$-калибровки при различных значениях $a$. Для построения численных решений в четном по твисту универсальном пространстве использованы приближение за счет отсечения уровней и итерационная процедура. Найденные калибровочные инварианты численно устойчивы и практически равны соответствующим инвариантам решения Шнабля для конденсации тахионов. Полученные результаты служат дополнительным свидетельством в пользу того, что эти численные и аналитическое решения калибровочно-эквивалентны.
\end{abstract}

Ключевые слова: струнная теория поля, конденсация тахионов, калибровочно-инвариантное перекрытие.

\section{1. ВВЕДЕНИЕ}

Считается, что струнная теория поля является кандидатом на непертурбативную формулировку теории струн. Исследование решений струнных теорий поля важно для понимания непертурбативных явлений в теории струн. В частности, после того, как Шнабль построил аналитическое решение [1] для конденсации тахионов в рамках кубичной теории поля открытых бозонных струн, в этой области получено множество новых результатов.

Важное свойство решения Шнабля выражается высотой потенциала на решении, которая определяется путем вычисления действия и относительно которой аналитически доказано ее равенство натяжению D25-браны в согласии с гипотезой Сена. Другая калибровочно-инвариантная наблюдаемая, называемая калибровочно-инвариантным перекрытием, была вычислена для решения Шнабля с помощью аналитического метода [2], [3], а также в рамках приближения (отсечения) по $L_{0}$-уровням [3].

${ }^{*}$ Theoretical Physics Laboratory, RIKEN, Wako, Japan. E-mail: ikishimo@riken.jp

${ }^{\dagger}$ Department of Physics, Nara Women's University, Nara, Japan.

E-mail: tomo@asuka.phys.nara-wu.ac.jp 
Полученное значение доказывает, что решение может быть связано с граничным состоянием для D-браны [2], [4].

C другой стороны, еще до получения аналитического решения Шнабля было построено численное решение в калибровке Зигеля с использованием приближения за счет отсечения уровней, причем была вычислена высота его потенциала [5]-[7]. Она оказалась практически такой же, как и натяжение D-браны. B работе [3] было вычислено калибровочно-инвариантное перекрытие для численного решения, и оказалось, что его значение почти такое же, как и для аналитического решения. Эти результаты согласуются с предполагаемой калибровочной эквивалентностью этих решений.

В действительности имеются и другие численные решения. В настоящей работе мы сконцентрируем наше внимание на решениях в $a$-калибровке Асано-Като [8], предложенной как условие, непротиворечиво фиксирующее калибровку и зависящее от вещественного параметра $a$, соответствующего ковариантной калибровке в стандартной калибровочной теории. С использованием такой калибровки были построены численные решения для конденсации тахионов, а в работе [9] для них были вычислены высоты потенциалов в приближении за счет отсечения уровней вплоть до уровня $(6,18)$. Нами построены численные решения в $a$-калибровке при различных $a$ для высших уровней и вычислены калибровочные инварианты, действие и калибровочно-инвариантное перекрытие [10].

Оказалось, что при увеличении уровня отсечения значения для каждой конфигурации приближаются к соответствующим значениям, полученным из аналитического решения. Отсюда следует, что все эти численные решения в различных $a$-калибровках, а не только в калибровке Зигеля, калибровочно-эквивалентны решению Шнабля, полученному аналитически. А именно, эти различные решения могут представлять единый непертурбативный тахионный вакуум в теории поля бозонных струн. Более того, наши численные результаты могут означать, что для исследования непертурбативного вакуума в теории поля бозонных струн приближение за счет отсечения уровней может быть надежным методом не только в калибровке Зигеля, но и в $a$-калибровке.

Работа построена следующим образом. Прежде всего, мы кратко напоминаем, что такое калибровочно-инвариантное перекрытие (раздел 2 ) и условие $a$-калибровки (раздел 3). Затем в разделе 4 мы объясняем наш метод построения численных решений. В разделе 5 приводятся полученные численные результаты. Наконец, раздел 6 представляет собой заключение.

\section{2. КАЛИБРОВОЧНО-ИНВАРИАНТНОЕ ПЕРЕКРЫТИЕ}

Калибровочно-инвариантное перекрытие $\mathcal{O}_{V}(\Psi)$ определяется как свертка струнного поля открытой струны $\Psi$ и некоторого состояния замкнутой струны на массовой оболочке. Более точно, $\mathcal{O}_{V}(\Psi)$ можно выразить как ${ }^{1)}$

$$
\mathcal{O}_{V}(\Psi)=\left\langle\hat{\gamma}\left(1_{\mathrm{c}}, 2\right) \mid \Phi_{V}\right\rangle_{1_{\mathrm{c}}}|\Psi\rangle_{2}
$$

1) Подробности см., например, в работе [3]. 
где $\left\langle\hat{\gamma}\left(1_{\mathrm{c}}, 2\right)\right|$ - вершина Шапиро-Торна [11], а $\left|\Phi_{V}\right\rangle$ задается примарным полем материи $V_{\mathrm{m}}(z, \bar{z})$ с размерностью $(1,1):\left|\Phi_{V}\right\rangle=c_{1} \bar{c}_{1} V_{\mathrm{m}}(0,0)|0\rangle$. Используя соотношения $\mathcal{O}_{V}\left(Q_{\mathrm{B}} \Lambda\right)=0$ и $\mathcal{O}_{V}(\Psi * \Lambda)=\mathcal{O}_{V}(\Lambda * \Psi)$, можно заметить, что $\mathcal{O}_{V}(\Psi)$ является калибровочно-инвариантным: $\delta_{\Lambda} \mathcal{O}_{V}(\Psi)=0$ под действием калибровочного преобразования струнного поля $\delta_{\Lambda} \Psi=Q_{\mathrm{B}} \Lambda+\Psi * \Lambda-\Lambda * \Psi$, которое оставляет инвариантным действие

$$
S(\Psi)=-\frac{1}{g^{2}}\left(\frac{1}{2}\left\langle\Psi, Q_{\mathrm{B}} \Psi\right\rangle+\frac{1}{3}\langle\Psi, \Psi * \Psi\rangle\right) .
$$

Вычислим калибровочно-инвариантное перекрытие для решения Шнабля в случае конденсации тахионов $\Psi_{\mathrm{Sch}}[1]$, которое можно выразить как

$$
\Psi_{\mathrm{Sch}}=\psi_{0}+\sum_{n=0}^{\infty}\left(\psi_{n+1}-\psi_{n}-\left.\partial \psi_{r}\right|_{r=n}\right)
$$

с конкретным струнным полем $\psi_{r}$ вида

$$
\psi_{r}=\frac{2}{\pi} U_{r+2}^{\dagger} U_{r+2}\left[-\frac{1}{\pi}\left(\mathcal{B}_{0}+\mathcal{B}_{0}^{\dagger}\right) \tilde{c}\left(\tilde{x}_{r}\right) \tilde{c}\left(-\tilde{x}_{r}\right)+\frac{1}{2}\left(\tilde{c}\left(\tilde{x}_{r}\right)+\tilde{c}\left(-\tilde{x}_{r}\right)\right)\right]|0\rangle,
$$

где $\tilde{x}_{r}=\pi r / 4, U_{r}=(2 / r)^{\mathcal{L}_{0}}, \mathcal{B}_{0}=b_{0}+\sum_{k=1}^{\infty}\left(2(-1)^{k+1} /\left(4 k^{2}-1\right)\right) b_{2 k}, \mathcal{L}_{0}=\left\{Q_{\mathrm{B}}, \mathcal{B}_{0}\right\}$ и $\tilde{c}(\tilde{z})=(\cos \tilde{z})^{2} c(\operatorname{tg} \tilde{z})$. Пользуясь тем, что $\mathcal{O}_{V}\left(\psi_{r}\right)$ не зависит от $r$, получаем [2]-[4]

$$
\mathcal{O}_{V}\left(\Psi_{\mathrm{Sch}}\right)=\mathcal{O}_{V}\left(\psi_{0}\right)=\frac{1}{2 \pi}\left\langle B\left|c_{0}^{-}\right| \Phi_{V}\right\rangle
$$

где $\langle B|$ - граничное состояние для D-браны. Чтобы получить ненулевое значение для полей открытой струны с нулевым импульсом, в качестве состояния на массовой оболочке возьмем дилатонное состояние с нулевым импульсом: $\Phi_{V}=$ $-(1 / 26) \eta_{\mu \nu} \alpha_{-1}^{\mu} \bar{\alpha}_{-1}^{\nu} c_{1} \bar{c}_{1}|0\rangle$. Тогда из формулы (5) получаем

$$
\frac{\mathcal{O}_{V}\left(\Psi_{\text {Sch }}\right)}{V_{26}}=\frac{1}{2 \pi}
$$

где $V_{26}$ - объемный множитель.

\section{3. а-КАЛИБРОВКА АСАНО-КАТО}

Предложенное в работе [8] условие $a$-калибровки в классическом секторе, а именно в секторе с равным единице духовым числом на мировом листе ${ }^{2)}$, определяется как

$$
\left(b_{0} M+a b_{0} c_{0} \widetilde{Q}\right) \Phi_{1}=0 .
$$

Здесь $a$ - вещественный параметр, а операторы $M$ и $\widetilde{Q}$ определяются через разложе-

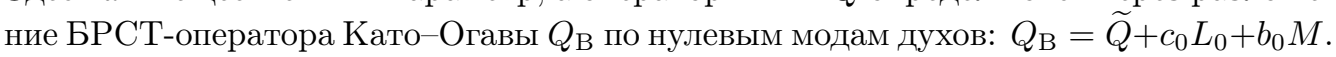
В случае $a=0$ условие (7) эквивалентно стандартной калибровке Фейнмана-Зигеля. Действительно, исследуя безмассовый сектор на уровне квадратичного действия для

\footnotetext{
2) Условия $а$-калибровки для секторов с любым духовым числом явно указаны в работах [8], [12].
} 
струнного поля с учетом пространственно-временных духовых полей, можно заметить, что параметр $a$ соответствует калибровочному параметру $\alpha$ в ковариантной калибровке в случае обыкновенной калибровочной теории согласно $\alpha=1 /(a-1)^{2}$ [8]. При $a=\infty$ условие $(7)$ имеет вид $b_{0} c_{0} \widetilde{Q} \Phi_{1}=0$ и соответствует калибровке Ландау.

Следует отметить, что в случае $a=1$ условие (7) некорректно определено в свободной теории, поскольку оно принимает вид $b_{0} c_{0} Q_{\mathrm{B}} \Phi_{1}=0$, а таким способом нельзя пертурбативно зафиксировать калибровку.

\section{4. ПОСТРОЕНИЕ ЧИСЛЕННЫХ РЕШЕНИЙ}

В этом разделе мы объясним наш метод построения численных решений в $a$-калибровке. Используем итерационную процедуру, которая применялась в случае калибровки Зигеля в работе [7]. В качестве исходной конфигурации $\Psi_{(0)}$ возьмем

$$
\Psi_{(0)}=\frac{64}{81 \sqrt{3}} c_{1}|0\rangle
$$

что представляет собой единственное нетривиальное решение в приближении самого низшего уровня в $а$-калибровке. Далее, если уже имеется $\Psi_{(n)}$, то зададим следующую конфигурацию $\Psi_{(n+1)}$ как решение линейных уравнений

$$
\begin{gathered}
\left(b_{0} M+a b_{0} c_{0} \widetilde{Q}\right) \Psi_{(n+1)}=0, \\
\mathcal{P}\left(Q_{\Psi_{(n)}} \Psi_{(n+1)}-\Psi_{(n)} * \Psi_{(n)}\right)=0,
\end{gathered}
$$

где

$$
Q_{\Psi_{(n)}} \Phi \equiv Q_{\mathrm{B}} \Phi+\Psi_{(n)} * \Phi-(-1)^{|\Phi|} \Phi * \Psi_{(n)} .
$$

Первое уравнение представляет собой условие $a$-калибровки для $\Psi_{(n+1)}$, а второе получается из уравнения движения

$$
Q_{\mathrm{B}} \Psi+\Psi * \Psi=0 \text {. }
$$

$\mathcal{P}$ представляет собой подходящий проекционный оператор для решения этих уравнений. В численных расчетах мы для простоты выбираем $\mathcal{P}=c_{0} b_{0}$. Если приведенная выше итерация сходится к некоторой конфигурации $\Psi_{(\infty)}$, то последняя удовлетворяет условию $a$-калибровки и

$$
\mathcal{P}\left(Q_{\mathrm{B}} \Psi_{(\infty)}+\Psi_{(\infty)} * \Psi_{(\infty)}\right)=0
$$

что представляет собой спроектированную часть уравнения движения. Чтобы подтвердить полное уравнение движения (12) для сходящейся конфигурации, следует проверить остающуюся часть ${ }^{3)}$

$$
(1-\mathcal{P})\left(Q_{\mathrm{B}} \Psi_{(\infty)}+\Psi_{(\infty)} * \Psi_{(\infty)}\right)=0,
$$

где в нашем случае $1-\mathcal{P}=b_{0} c_{0}$.

\footnotetext{
${ }^{3)}$ В работе [13] это условие в калибровке Зигеля названо БРСТ-инвариантностью и исследовано для численного решения.
} 
В действительности мы выполнили приведенную выше процедуру численно для стандартного приближения за счет отсечения уровней. Мы построили численное решение в $a$-калибровке при различных $a$ для уровней, отсекаемых по $(L, 2 L)$ и $(L, 3 L)$, где $L$ обозначает максимальный уровень (собственное значение $\left.L_{0}+1\right)$ для усеченного струнного поля, а $2 L$ или $3 L$ указывает максимальный полный уровень, оставляемый в членах с трехструнным взаимодействием. Начав с уравнения (8), мы продолжаем описанную выше итерационную процедуру до тех пор, пока относительная ошибка не достигнет значения $\left\|\Psi_{(M)}-\Psi_{(M-1)}\right\| /\left\|\Psi_{(M)}\right\|<10^{-8}$, где $\|(\cdots)\|$ обозначает евклидову норму по отношению к ортонормированному базису. Далее выясняется, что для найденной конфигурации выполнено условие $\| \mathcal{P}\left(Q_{\mathrm{B}} \Psi_{(M)}+\right.$ $\left.\Psi_{(M)} * \Psi_{(M)}\right)\|/\| \Psi_{(M)} \|<10^{-8}$. Оказалось, что для различных $a$ (за исключением опасной области $a \sim 1$, лежащей вблизи калибровочного условия, которое некорректно определено в рамках теории возмущений, как отмечалось в разделе 3) конфигурация достигает указанной точности после десяти или даже меньшего числа итерационных шагов.

Для каждой полученной сходящейся конфигурации мы вычислили левую часть выражения (14) и проверили, что различные коэффициенты стремятся к нулю, а величина

$$
\frac{\left\|(1-\mathcal{P})\left(Q_{\mathrm{B}} \Psi_{(M)}+\Psi_{(M)} * \Psi_{(M)}\right)\right\|}{\left\|\Psi_{(M)}\right\|}
$$

также обращается в нуль при увеличении уровня усечения. Поэтому мы рассматривали полученные нами конфигурации как численные решения в $a$-калибровке для полного уравнения движения (12) и вычислили для них калибровочные инварианты, действие и калибровочно-инвариантное перекрытие.

\section{5. ВЫЧИСЛЕНИЕ КАЛИБРОВОЧНЫХ ИНВАРИАНТОВ}

\section{1. Калибровочные инварианты для численных решений в калибровке}

Зигеля. Для численного решения в калибровке Зигеля $b_{0} \Psi=0$, которая в терминах $a$-калибровки соответствует случаю $a=0$, вычисления проводить легче, чем для других значений $a$. Мы провели численные расчеты вплоть до уровня $L=20^{4}$.

Полученные в настоящей работе численные результаты приведены в табл. 1 и 2 . Как следует из табл. 1, значения действия превышают $100 \%$ натяжения D-браны при $L \geqslant 14$. Это явление было замечено ранее, причем выражалась надежда, что получаемые значения вернутся к единице для более высоких уровней [7]. С другой стороны, как следует из табл. 2, значения калибровочно-инвариантного перекрытия монотонно приближаются к аналитическому значению, соответствующему решению Шнабля, хотя скорость приближения достаточно невелика по сравнению с поведением действия.

Как бы то ни было, из наших результатов, приведенных в табл. 1 и 2, видимо, следует, что полученные (нормированные) калибровочные инварианты при $L \rightarrow \infty$ стремятся к единице. Если это так, то это свидетельствует о калибровочной эквивалентности между численным решением в калибровке Зигеля и аналитическим решением Шнабля [3].

\footnotetext{
4) Вычисления для высших значений уровня отсечения $(L \geqslant 18)$ проводились с помощью нашей программы на $\mathrm{C}++$ и Фортране.
} 
ТАБлицА 1. Значения действия для численного решения при отсечении уровней по $(L, 2 L)$ и $(L, 3 L)$ в калибровке Зигеля. Значения нормированы на соответствующий аналитический результат для решения Шнабля $S\left(\Psi_{\mathrm{Sch}}\right) / V_{26}=1 /\left(2 \pi^{2} g^{2}\right)$, который равен натяжению D-браны. Вплоть до уровня $L=18$ приведенные данные согласуются с результатами работы [7].

\begin{tabular}{|c|c|c|}
\hline$L$ & $\left.2 \pi^{2} g^{2} S(\Psi)\right|_{(L, 2 L)} / V_{26}$ & $\left.2 \pi^{2} g^{2} S(\Psi)\right|_{(L, 3 L)} / V_{26}$ \\
\hline 2 & 0.948553 & 0.959377 \\
\hline 4 & 0.986403 & 0.987822 \\
\hline 6 & 0.994773 & 0.995177 \\
\hline 8 & 0.997780 & 0.997930 \\
\hline 10 & 0.999116 & 0.999182 \\
\hline 12 & 0.999791 & 0.999822 \\
\hline 14 & 1.000158 & 1.000174 \\
\hline 16 & 1.000368 & 1.000375 \\
\hline 18 & 1.000490 & 1.000494 \\
\hline 20 & 1.000562 & 1.000563 \\
\hline
\end{tabular}

ТАБлицА 2. Значения калибровочно-инвариантного перекрытия для численного решения при отсечении уровней по $(L, 2 L)$ и $(L, 3 L)$ в калибровке Зигеля. Значения нормированы на соответствующий аналитический результат для решения Шнабля (6).

\begin{tabular}{|c|c|c|}
\hline$L$ & $2 \pi \mathcal{O}_{V}\left(\Psi_{(L, 2 L)}\right) / V_{26}$ & $2 \pi \mathcal{O}_{V}\left(\Psi_{(L, 3 L)}\right) / V_{26}$ \\
\hline 2 & 0.878324 & 0.889862 \\
\hline 4 & 0.929479 & 0.931952 \\
\hline 6 & 0.950175 & 0.951079 \\
\hline 8 & 0.960617 & 0.961175 \\
\hline 10 & 0.967790 & 0.968115 \\
\hline 12 & 0.972321 & 0.972560 \\
\hline 14 & 0.976005 & 0.976171 \\
\hline 16 & 0.978544 & 0.978677 \\
\hline 18 & 0.980802 & 0.980904 \\
\hline 20 & 0.982432 & 0.982517 \\
\hline
\end{tabular}




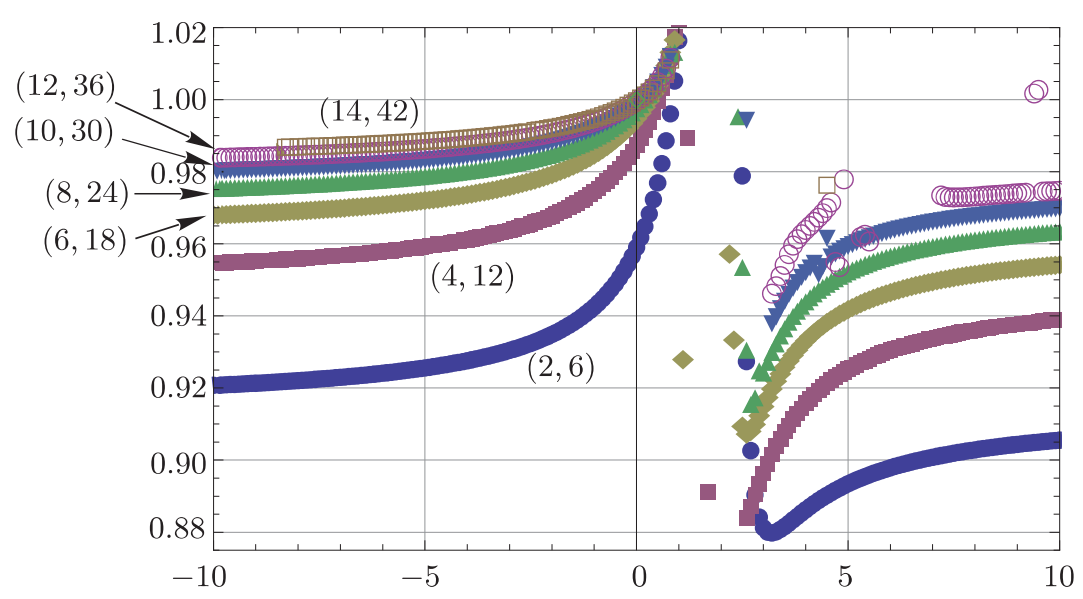

Рис. 1. Графики действия для различных решений $\Psi_{a, L}$ в $a$-калибровке при отсечении уровней по $(L, 3 L)$. По горизонтальной оси отложены значения $a$, по вертикальной - значения нормированного действия $2 \pi^{2} g^{2} S\left(\Psi_{a, L}\right) / V_{26}$. Пометка $(L, 3 L)$ для каждой "кривой” указывает уровень отсечения.

\section{2. Калибровочные инварианты для численных решений в $a$-калиб-} ровке. В этом разделе мы вычислим калибровочные инварианты для численных решений в $a$-калибровке. На рис. $1-3$ приведены результаты для отсечения на уровне $(L, 3 L)^{5)}$. Аналогичные результаты получаются в случае отсечения по $(L, 2 L)$ [10].

Для различных $a$ в областях $a \lesssim 0, a \gg 1$ нормированные калибровочные инварианты, действие (рис. 1) и калибровочно-инвариантное перекрытие (рис. 2) приближаются к единице при увеличении уровня. Скорость приближения к единице для калибровочно-инвариантного перекрытия меньше, чем соответствующая скорость для действия, как в случае калибровки Зигеля (см. табл. 1, 2). Хотя только $a=1$-калибровка определена некорректно в свободной теории, взаимодействия включены в численные вычисления, а потому значения в области $a \sim 1$ являются неустойчивыми. В действительности итерации не сходятся в опасной области $a \sim 1$.

Из рис. 3 видно, что оба (нормированных) калибровочных инварианта в случае численных решений в различных $a$-калибровках выражают тенденцию стремиться к единице при увеличении уровня отсечения. А именно, в пределе $L \rightarrow \infty$ имеем свидетельства в пользу поведения

$$
S\left(\Psi_{a, L}\right) \rightarrow S\left(\Psi_{\mathrm{Sch}}\right), \quad \mathcal{O}_{V}\left(\Psi_{a, L}\right) \rightarrow \mathcal{O}_{V}\left(\Psi_{\mathrm{Sch}}\right)
$$

при различных $a(-\infty \leqq a \lesssim 0,1 \ll a \leqq \infty)$. Видимо, отсюда следует, что не только решение в калибровке Зигеля $(a=0)$, но и все различные решения в $a$-калибровках, построенные аналогично тому, как это было сделано в разделе 4 , калибровочно-эквивалентны аналитическому решению Шнабля.

\footnotetext{
5) Только одно значение было получено в калибровке Зигеля $(a=0)$ для отсечения на уровне $(16,48)$. Для других $a$-калибровок $(a \neq 0)$ вычисления, которые мы проводим с помощью программы Mathematica, являются более сложными.
} 


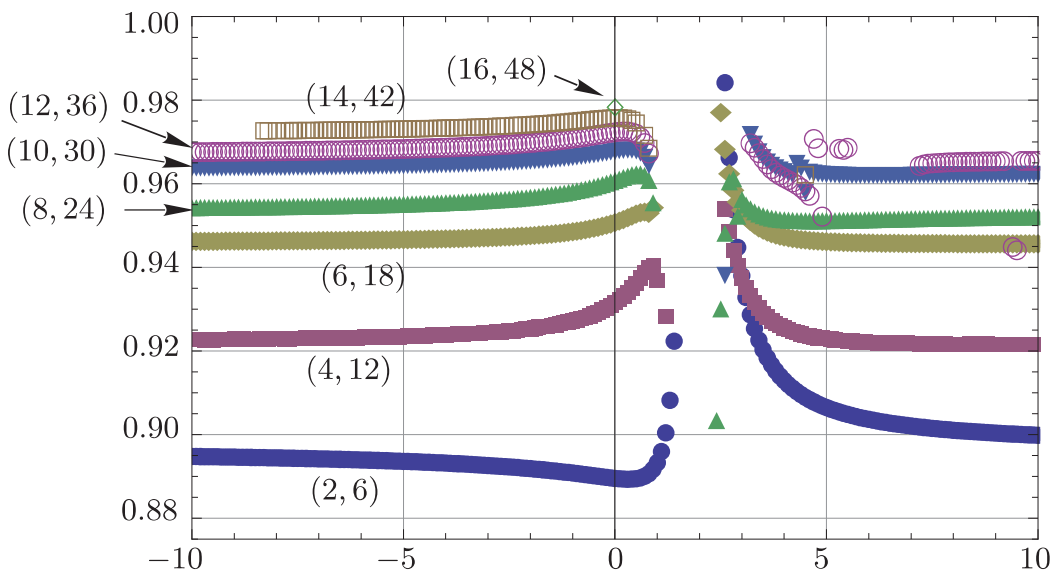

Рис. 2. Графики калибровочно-инвариантного перекрытия для различных решений $\Psi_{a, L}$ в $a$-калибровке при отсечении по $(L, 3 L)$. По горизонтальной оси отложены значения $a$, по вертикальной - нормированное калибровочно-инвариантное перекрытие $2 \pi \mathcal{O}_{V}\left(\Psi_{a, L}\right) / V_{26}$.

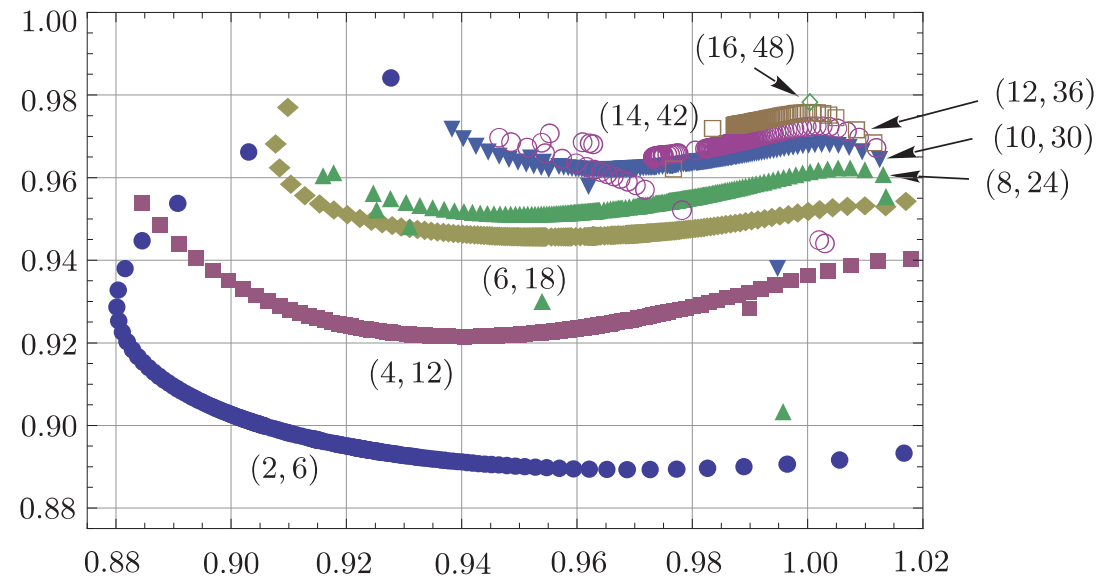

Рис. 3. Графики калибровочных инвариантов для различных решений $\Psi_{a, L}$ в $a$-калибровке при отсечении по $(L, 3 L)$. По горизонтальной оси отложено нормированное действие, по вертикальной - нормированное калибровочно-инвариантное перекрытие. Каждой точке соответствует значение величины $\left(2 \pi^{2} g^{2} S\left(\Psi_{a, L}\right) / V_{26}, 2 \pi \mathcal{O}_{V}\left(\Psi_{a, L}\right) / V_{26}\right)$ для различных $a$, включая $a=\infty$. Левая часть "кривой" для каждого уровня соответствует области $4 \lesssim a<+\infty$, а правая - области $-\infty<a \lesssim 1 / 2$. Графики для $a \rightarrow+\infty$ и $a \rightarrow-\infty$ непрерывно связаны через график для калибровки Ландау $(a=\infty)$. 


\section{6. ЗАКЛЮЧЕНИЕ}

Мы вычислили калибровочные инварианты (действие и калибровочно-инвариантное перекрытие) для численных решений в $a$-калибровке в рамках отсечения уровней. Для построения этих решений мы использовали метод итераций и проверили для них уравнения движения. Кроме области, в которой $a \sim 1$, где условие $a$-калибровки становится некорректно определенным в свободной теории, все наши решения в $a$-калибровке воспроизводят аналитические значения для конденсации тахионов в решении Шнабля. Результаты согласуются с ожидаемой калибровочной эквивалентностью различных решений в $a$-калибровке, включая решение в калибровке Зигеля $(a=0)$ и решения Шнабля. Поэтому они могут представлять единый непертурбативный вакуум, где D25-брана исчезает.

Благодарности. Настоящая работа была выполнена при поддержке JSPS Grantin-Aid for Scientific Research (C) (\#21540269). Работа И. Кишимото была поддержана со стороны Special Postdoctoral Researchers Program at RIKEN. Работа T. Такахаши была частично поддержана за счет Nara Women's University Intramural Grant for Project Research. Численные расчеты в настоящей работе частично были проведены с помощью Компьютерного центра Института теоретической физики Юкавы в университете Киото и в RIKEN Integrated Cluster of Clusters (RICC) facility.

\section{Список литературы}

[1] M. Schnabl, Adv. Theor. Math. Phys., 10:4 (2006), 433-501; arXiv: hep-th/0511286.

[2] I. Ellwood, JHEP, 08 (2008), 063; arXiv: 0804.1131.

[3] T. Kawano, I. Kishimoto, T. Takahashi, Nucl. Phys. B, 803:1-2 (2008), 135-165; arXiv: 0804.1541.

[4] T. Kawano, I. Kishimoto, T. Takahashi, Phys. Lett. B, 669:5 (2008), 357-358; arXiv: 0804.4414.

[5] A. Sen, B. Zwiebach, JHEP, 03 (2000), 002; arXiv: hep-th/9912249.

[6] N. Moeller, W. Taylor, Nucl. Phys. B, 583:1-2 (2000), 105-144; arXiv: hep-th/0002237.

[7] D. Gaiotto, L. Rastelli, JHEP, 08 (2003), 048; arXiv: hep-th/0211012.

[8] M. Asano, M. Kato, Progr. Theoret. Phys., 117:3 (2007), 569-587; arXiv: hep-th/0611189.

[9] M. Asano, M. Kato, JHEP, 01 (2007), 028; arXiv: hep-th/0611190.

[10] I. Kishimoto, T. Takahashi, Progr. Theoret. Phys., 121:4 (2009), 695-710; arXiv: 0902.0445.

[11] J. A. Shapiro, C. B. Thorn, Phys. Lett. B, 194:1 (1987), 43-48.

[12] M. Asano, M. Kato, Nucl. Phys. B, 807:1-2 (2009), 348-372; arXiv: 0807.5010.

[13] H. Hata, S. Shinohara, JHEP, 09 (2000), 035; arXiv: hep-th/0009105. 